\title{
Precisão experimental de ensaios de feijão analisada pelo método de Papadakis
}

\author{
Lindolfo Storck ${ }^{(1)}$, Nerinéia Dalfollo Ribeiro(1) e Alberto Cargnelutti Filho(1)
}

(1)Universidade Federal de Santa Maria, Departamento de Fitotecnia, Avenida Roraima, № 1.000, Bairro Camobi, CEP $97105-900$ Santa
Maria, RS. E-mail: lindolfo@pq.cnpq.br, nerineia@pq.cnpq.br, cargnelutti@pq.cnpq.br

Resumo - O objetivo deste trabalho foi verificar se o uso do método de Papadakis na análise dos resultados dos ensaios de competição de cultivares de feijão modifica os pressupostos do modelo matemático e as medidas de precisão experimental. Foram utilizados dados de produtividade de grãos de feijão de 26 ensaios de competição de cultivares, conduzidos em delineamento de blocos ao acaso. Para cada ensaio, sem e com uso do método de Papadakis, foram realizados verificação dos pressupostos, análise de variância e testes de hipóteses, e calculadas as estatísticas para identificação da precisão experimental. Os pressupostos não foram violados por nenhum dos métodos de análise (sem e com Papadakis). O índice de diferenciação de Fasoulas aumentou de 5,9 para 13,9, e a acurácia seletiva de 0,82 para 0,89, com uso do método de Papadakis. Esse método permitiu que se obtivesse $80 \%$ de precisão na identificação de cultivares de feijão superiores quanto à produtividade de grãos com uso de apenas quatro repetições; enquanto que, na análise sem uso de Papadakis, foram necessárias sete repetições para manter a precisão de $80 \%$. O método de Papadakis melhora os índices das medidas de precisão experimental e reduz o número de repetições necessário para a predição do desempenho de cultivares de feijão, em termos de produtividade de grãos.

Termos para indexação: Phaseolus vulgaris, análise espacial, ensaios de competição, medidas de precisão, pressupostos.

\section{Experimental precision in common bean trials using the Papadakis method}

\begin{abstract}
The objective of this work was to verify whether the use of the Papadakis method in the analysis of the results of common bean cultivar trials modifies the assumptions of the mathematical model and the experimental precision indicators. Common bean yield data obtained from 26 cultivar trials carried out in randomized complete block design were used. For each trial, with or without the use of the Papadakis method, the assumptions were verified, the analysis of variance and the hypothesis tests were carried out, and statistics were calculated in order to identify experimental precision. The assumptions were not violated by either method (with or without the Papadakis method). The Fasoulas index increased from 5.9 to 13.9, and the selective accuracy from 0.82 to 0.89 with the Papadakis method. This method allowed the identification, with $80 \%$ precision, of superior common bean cultivars regarding grain yield using only four replicates, while seven replicates were necessary to maintain $80 \%$ precision in the analysis without the Papadakis method. The Papadakis method improves the indices of experimental precision and reduces the number of replicates necessary to predict the performance of common bean cultivars regarding grain yield.
\end{abstract}

Index terms: Phaseolus vulgaris, spatial analysis, competition trials, precision indicators, assumptions.

\section{Introdução}

Erros de identificação da superioridade de uma cultivar, decorrentes da insuficiência de precisão experimental dos ensaios, repercutem na produtividade da espécie cultivada, pois os resultados desses ensaios são usados para a inscrição das novas cultivares no Registro Nacional de Cultivares, no Ministério da Agricultura, Pecuária e Abastecimento (RNC-MAPA). Além disso, os resultados gerados pela rede de ensaios têm fornecido informações sobre o desempenho das cultivares para os órgãos de assistência técnica.

Conhecer a área e o material experimental, para determinação adequada do delineamento experimental, do número de repetições e do tamanho de parcelas, e manter a uniformidade do manejo são procedimentos indispensáveis para a execução de ensaios com precisão experimental de magnitude aceitável (Gómez \& Gómez, 1984; Steel et al., 1997; Ramalho et al., 2005; Banzatto \& Kronka, 2006). 
Medidas adequadas de avaliação da precisão também são importantes. Nesse sentido, as estatísticas acurácia seletiva, coeficiente de herdabilidade, coeficiente de determinação e valor do teste $\mathrm{F}$ para cultivares têm relação direta entre si e são mais adequadas do que o coeficiente de variação e a diferença mínima significativa, para avaliar a precisão experimental em ensaios de competição de híbridos de milho (Zea mays L.) (Cargnelutti Filho \& Storck, 2007, 2009). Ao avaliar dados da produtividade de ensaios de competição de cultivares de feijão (Phaseolus vulgaris L.) e de soja (Glycine max L.), Cargnelutti Filho et al. (2009b), concluíram que os limites de classe de precisão estabelecidos para cultivares, a partir das estatísticas acurácia seletiva, coeficiente de herdabilidade, coeficiente de determinação e valor do teste $\mathrm{F}$, são adequados. Melhorias na precisão experimental, em ensaios de feijão, também foram investigadas quanto ao tamanho da amostra de caracteres das plantas (Cargnelutti Filho et al., 2008), ao uso de bordaduras nas parcelas (Ribeiro et al., 2001) e ao número de repetições (Cargnelutti Filho et al., 2009a).

Entre as formas de análise espacial (Costa et al., 2005), o método de Papadakis (Papadakis, 1937) consiste no ajuste dos valores obtidos nas parcelas em função das médias dos erros experimentais entre as parcelas vizinhas, usadas como covariável, com a finalidade de diminuir a variância do erro experimental. A covariável mais adequada é a estimada em função do erro das parcelas de referência e das mais próximas (lado esquerdo, direito, frente e fundo) (Cargnelutti Filho et al., 2003).

O uso do método de Papadakis em 226 ensaios de soja, com diversidade de ambientes e de recursos genéticos, mostrou-se eficiente para a melhoria dos indicadores de precisão experimental (Storck et al., 2008). Esse método, conforme Cargnelutti Filho et al. (2003), proporcionou ganhos de precisão em um estudo com cinco ensaios com milho e, posteriormente, com 25 ensaios com híbridos de milho (Storck et al., 2010). A magnitude da eficiência do método de Papadakis e a sua influência sobre os pressupostos da análise de variância (normalidade e independência do erro, homogeneidade de variância entre cultivares e aditividade do modelo), para ensaios com diferentes cultivares de feijão, executados durante uma sequência de anos em uma mesma área experimental, ainda não são conhecidas. No entanto, de acordo com Candido et al. (2009), o método de Papadakis não melhora a precisão experimental nem as sequências no ordenamento de genótipos de cana-de-açúcar (Saccharum officinarum L.), em termos de potencial produtivo (colmos), provavelmente por se tratar de uma característica que não depende tanto da interação com as condições ambientais, em comparação com a produção dos grãos.

O objetivo deste trabalho foi verificar se o uso do método de Papadakis na análise dos resultados dos ensaios de competição de cultivares de feijão modifica os pressupostos do modelo matemático e os indicadores de precisão experimental.

\section{Material e Métodos}

Foram utilizados dados de produtividade de grãos de feijão obtidos em 26 ensaios de competição de 13 a 26 cultivares por ensaio, no período de safra e de safrinha dos anos agrícolas de 1998/1999 a 2008/2009. Todos os ensaios foram conduzidos na mesma área experimental, do Departamento de Fitotecnia, da Universidade Federal de Santa Maria, RS $\left(29^{\circ} 42^{\prime} \mathrm{S}\right.$ e $53^{\circ} 49^{\prime} \mathrm{W}$, a $95 \mathrm{~m}$ de altitude), no delineamento de blocos ao acaso, com três ou quatro repetições. As unidades experimentais foram compostas de quatro fileiras de 4,0 m de comprimento, espaçadas em 0,50 m, e de área útil de 3,0 $\mathrm{m}^{2}$.

Os dados de produtividade de grãos, de cada ensaio, foram submetidos à análise de variância, com testes de hipóteses a $5 \%$ de probabilidade, para blocos e cultivares. Foram estimadas a eficiência relativa (ER) do uso de blocos, a média por cultivar, a média geral ( $(\hat{\mathrm{m}})$ e a amplitude entre as médias das cultivares. Também foram estimados o coeficiente de variação $(\mathrm{CV})$ e a diferença mínima significativa entre as cultivares, pelo teste de Tukey, a 5\% de probabilidade (D), calculada por:

$$
\left.\mathrm{D}=\mathrm{q}_{(\alpha ; \mathrm{I} ; \mathrm{GL}}\right) \sqrt{\mathrm{QM}_{\mathrm{E}} / \mathrm{J}}
$$

em que: $\mathrm{q}_{\left(\alpha ; \mathrm{I} ; \mathrm{GL} \mathrm{L}_{\mathrm{E}}\right)}$ é o valor da Tabela para o teste de Tukey, I é o número de cultivares, $\mathrm{GL}_{\mathrm{E}}$ são os graus de liberdade do erro, $\mathrm{QM}_{\mathrm{E}}$ é a estimativa do erro experimental e J é o número de repetições (blocos). A diferença mínima significativa entre as cultivares, pelo teste de Tukey, expressa em percentagem da média (DMS), foi obtida por: $\mathrm{DMS}=100 \mathrm{D} / \hat{\mathrm{m}}$. Para avaliar a precisão experimental, foi obtido o índice de diferenciação de Fasoulas 
(IDF) (Fasoulas, 1983), estimado pela expressão:

$$
\mathrm{IDF}=200 \sum_{\mathrm{i}=1}^{\mathrm{n}} \mathrm{m}_{\mathrm{i}} /[\mathrm{n}(\mathrm{n}-1)]
$$

em que: $\mathrm{n}$ é o número de cultivares do ensaio e $\mathrm{m}_{\mathrm{i}}$ é o número de médias que a i-ésima cultivar supera estatisticamente, após aplicação do teste de Tukey.

Determinou-se o valor do coeficiente de determinação (Cargnelutti Filho \& Storck, 2007), expresso por $\mathrm{R}^{2}=\mathrm{QM}_{\mathrm{g}} /\left(\mathrm{QM}_{\mathrm{g}}+\mathrm{QM}_{\mathrm{E}}\right)$, em que: $\mathrm{QM}_{\mathrm{g}}$ é o quadrado médio de cultivar. A acurácia seletiva (AS) foi estimada como AS $=\left[(1-(1 / F)]^{1 / 2}\right.$, em que: $\mathrm{F}$ é o valor do teste F para a cultivar (Resende \& Duarte, 2007; Cargnelutti Filho \& Storck, 2009).

Tendo-se considerado as avaliações em cada bloco como medições realizadas no mesmo indivíduo (cultivar), estimou-se o coeficiente de correlação intraclasse para cultivares, ou coeficiente de repetibilidade (método da análise de variância):

$$
\hat{\rho}_{\mathrm{g}}=\hat{\sigma}_{\mathrm{g}} /\left(\hat{\sigma}^{2}+\hat{\sigma}_{\mathrm{g}}^{2}\right),
$$

em que: $\hat{\sigma}_{\mathrm{g}}^{2}=\left(\mathrm{QM}_{\mathrm{g}}-\mathrm{QM}_{\mathrm{E}}\right) / \mathrm{J}$ e $\hat{\sigma}^{2}=\mathrm{QM}_{\mathrm{E}}$. O número mínimo de medições (repetições $=$ blocos $=\mathrm{J}$ ) necessário para predizer o valor real dos indivíduos (cultivares), com base na determinação ou na precisão pré-estabelecida $\left(\mathrm{R}^{2}\right)$, foi obtido conforme Cruz \& Regazzi (1997), ou seja, o valor de J, para um dado $\mathrm{R}^{2}$, é: $\mathrm{J}\left(\mathrm{R}^{2}\right)=\left[\left(1-\hat{\rho}_{\mathrm{g}}\right) \mathrm{R}^{2}\right] /\left[\hat{\rho}_{\mathrm{g}}\left(1-\mathrm{R}^{2}\right)\right]$.

Foram estimados os erros de cada unidade experimental, tendo-se considerado o delineamento de blocos ao acaso (valor observado menos a média geral, menos os efeitos de bloco e de cultivar). Aplicou-se o teste de Lilliefors (Sprent \& Smeeton, 2007) para verificar o pressuposto de normalidade dos erros. Para o teste da aleatoriedade dos erros ou teste de sequência (Sprent \& Smeeton, 2007), o número de sequências de erros positivos e negativos seguiu um caminhamento pré-estabelecido. A estatística do teste da sequência, igual ao número de vezes em que é trocado um sinal no caminhamento estabelecido para as parcelas experimentais, foi testada pela aproximação da distribuição normal padrão. $\mathrm{O}$ teste de Bartlett (Steel et al., 1997) foi aplicado para verificar a homogeneidade das variâncias dos erros entre as cultivares. A aditividade do modelo matemático foi verificada pelo teste de não aditividade do modelo matemático de Tukey (Snedecor \& Cochran, 1989). As hipóteses das pressuposições (normalidade, aleatoriedade, homogeneidade e aditividade) foram testadas a $5 \%$ de probabilidade.

Os erros foram estimados, tendo-se considerado o delineamento inteiramente casualizado (valor observado menos a média geral e menos o efeito de cultivar). A partir desses valores, estimou-se o erro médio de cada unidade experimental como média entre o erro da unidade considerada e os erros das unidades experimentais vizinhas (lado direito, lado esquerdo, da frente e do fundo) existentes (Cargnelutti Filho et al., 2003). O valor do erro médio foi denominado de covariável, para fins da análise de covariância, segundo o delineamento inteiramente casualizado (Steel et al., 1997; Storck et al., 2008), o que corresponde à aplicação do método de Papadakis (1937).

Os valores da covariável foram submetidos à análise de variância, conforme o modelo do delineamento de blocos ao acaso, e testados quanto aos pressupostos da normalidade, da aleatoriedade e da homogeneidade das variâncias. A análise de covariância, com testes de hipóteses dos efeitos da covariável e de cultivar ajustados, foi realizada de acordo com Steel et al. (1997) e com o cálculo das estimativas de amplitude entre médias de cultivares ajustadas, CV, D, DMS, IDF, $\mathrm{R}^{2}, \mathrm{AS}, \hat{\rho}_{\mathrm{g}}$ e J( $\left.\mathrm{R}^{2}\right)$.

Também foi estimada a eficiência relativa do uso da covariável, em relação ao uso do delineamento de blocos ao acaso, e testadas as hipóteses dos pressupostos (normalidade, aleatoriedade e homogeneidade das variâncias para cultivares) referentes ao erro neste modelo, e do paralelismo entre as cultivares quanto à resposta da covariável (Seber, 1976). Para todos os cálculos, foi elaborado e compilado um programa específico com linguagem Pascal.

\section{Resultados e Discussão}

A média da produção de grãos nos 26 ensaios foi de $1,61 \mathrm{Mg} \mathrm{ha}^{-1}$, tendo variado entre 0,25 e $3,00 \mathrm{Mg} \mathrm{ha}^{-1}$ (Tabela 1). Esses resultados são indicativos das variações do ambiente e da relação de cultivares dos ensaios, pois cada cultivar é avaliada por um número variado de anos. Em 12 ensaios (46\%), o efeito de blocos foi significativo (Tabela 2).

A variação entre as cultivares, significativa em $88 \%$ dos ensaios, e a ocorrência de falhas nos pressupostos (Tabela 2) estão próximas às encontradas nos estudos de 307 ensaios com milho (Marques et al., 2000) e de 25 ensaios de competição de híbridos de milho 
(Storck et al., 2010), e não foram motivo de restrição para a interpretação dos resultados.

Em todos os ensaios, a covariável não foi significativamente dependente do efeito de cultivar (Tabela 2). Portanto, as diferenças entre os tratamentos independem dos valores da covariável, pré-requisito para proceder à análise de covariância e ajustar as médias de cultivares em função das médias da covariável (Steel et al., 1997). Os pressupostos da aleatoriedade e da homogeneidade das variâncias também foram adequados. Contudo, a falta de normalidade da covariável foi observada em dois ensaios $(7,7 \%)$, condição mais favorável do que a observada para ensaios com soja (Storck et al., 2008). Em relação à covariável, os blocos foram: heterogêneos em $50 \%$ dos ensaios; superior ao observado em $38,9 \%$ dos ensaios com soja (Storck et al., 2008); e inferior ao observado em $60 \%$ dos ensaios com milho (Storck et al., 2010).

A média da eficiência relativa do uso de blocos foi igual a $133,8 \%$ (Tabela 3), pois $46 \%$ dos ensaios apresentaram blocos heterogêneos. Nos ensaios com soja, Storck et al. (2008) observaram que a média da ER foi igual a $134,5 \%$ em apenas $28,8 \%$ dos ensaios com blocos heterogêneos. Para ensaios com milho, a ER foi de $126 \%$ para $48 \%$ dos ensaios com blocos heterogêneos (Storck et al., 2010).

A aplicação do método de Papadakis aumentou, de 88 para $96 \%$, a proporção de ensaios com efeito significativo de cultivares (Tabela 2). O efeito da covariável também foi significativo em $92 \%$ dos ensaios. $\mathrm{O}$ uso do método de Papadakis melhorou todas as medidas de precisão experimental e reduziu a média do CV de 20,3 para $16,3 \%$; a média da diferença mínima significativa entre as cultivares, pelo teste de Tukey, de 0,882 para $0,715 \mathrm{Mg} \mathrm{ha}^{-1}$; a média da diferença mínima significativa entre as cultivares, pelo teste de Tukey, expresso em percentagem da média (DMS), de 61,4 para 49,4\%; e aumentou a média da AS de 0,821 para 0,886; a média do IDF de 5,95 para 13,62 ; e o coeficiente de determinação $\left(R^{2}\right)$ do

Tabela 1. Ano de cultivo, número de repetições $(\mathrm{J})$ e de cultivares (I), média da produtividade de grãos de feijão (Mg ha-1), quadrados médios dos blocos $\left(\mathrm{QM}_{\mathrm{B}}\right)$, das cultivares $\left(\mathrm{QM}_{\mathrm{G}}\right)$ e do erro $\left(\mathrm{QM}_{\mathrm{E}}\right)$ sem uso do método de Papadakis em 26 ensaios de competição de cultivares de feijão.

\begin{tabular}{|c|c|c|c|c|c|c|c|}
\hline Ensaio & Ano & $\mathrm{J}$ & $\mathrm{I}$ & Produtividade & $\mathrm{QM}_{\mathrm{B}}$ & $\mathrm{QM}_{\mathrm{G}}$ & $\mathrm{QM}_{\mathrm{E}}$ \\
\hline 1 & $1998 / 1999$ & 3 & 20 & 2,904 & $0,05148^{\text {ns }}$ & $0,52621 *$ & 0,17753 \\
\hline 2 & $1999 / 2000$ & 3 & 20 & 3,005 & $1,02714^{*}$ & $0,39552 *$ & 0,12914 \\
\hline 3 & $2000 / 2001$ & 3 & 16 & 1,850 & $0,07462^{\text {ns }}$ & $0,52604^{*}$ & 0,10357 \\
\hline 4 & $2000 / 2001$ & 3 & 24 & 1,545 & $0,74403 *$ & $0,52348 *$ & 0,12951 \\
\hline 5 & 2001 & 3 & 16 & 0,916 & $0,20769^{*}$ & $0,28031 *$ & 0,04273 \\
\hline 6 & $2001 / 2002$ & 3 & 19 & 2,393 & $20,78953 *$ & $0,35861^{\mathrm{ns}}$ & 0,20441 \\
\hline 7 & $2001 / 2002$ & 3 & 18 & 1,432 & $0,09859^{\mathrm{ns}}$ & $0,52498^{*}$ & 0,14337 \\
\hline 8 & $2001 / 2002$ & 3 & 14 & 1,248 & $0,43505^{*}$ & $0,20669^{*}$ & 0,09294 \\
\hline 9 & 2002 & 3 & 19 & 0,713 & $0,17129 *$ & $0,16745^{*}$ & 0,03062 \\
\hline 10 & $2002 / 2003$ & 3 & 19 & 1,864 & $0,05106^{\mathrm{ns}}$ & $1,00763 *$ & 0,12212 \\
\hline 11 & $2002 / 2003$ & 3 & 18 & 0,494 & $0,07929 *$ & $0,10599 *$ & 0,02351 \\
\hline 12 & 2003 & 3 & 19 & 0,253 & $0,04550^{*}$ & $0,02856^{*}$ & 0,00769 \\
\hline 13 & $2003 / 2004$ & 3 & 19 & 1,987 & $0,11168^{\mathrm{ns}}$ & $0,15879^{*}$ & 0,05799 \\
\hline 14 & $2003 / 2004$ & 3 & 26 & 1,595 & $1,13647^{*}$ & $0,41306^{*}$ & 0,13379 \\
\hline 15 & $2003 / 2004$ & 3 & 16 & 2,313 & $0,13676^{\mathrm{ns}}$ & $0,15982^{\text {ns }}$ & 0,09626 \\
\hline 16 & 2004 & 3 & 20 & 0,902 & $0,19421 *$ & $0,10726^{*}$ & 0,03599 \\
\hline 17 & $2004 / 2005$ & 3 & 18 & 1,969 & $0,59450 *$ & $0,39190 *$ & 0,03119 \\
\hline 18 & $2004 / 2005$ & 4 & 16 & 1,706 & $0,02907^{\mathrm{ns}}$ & $0,31439 *$ & 0,06989 \\
\hline 19 & $2005 / 2006$ & 3 & 21 & 2,351 & $0,29838^{\mathrm{ns}}$ & $0,43708^{*}$ & 0,16527 \\
\hline 20 & $2005 / 2006$ & 4 & 16 & 1,750 & $0,08660^{\mathrm{ns}}$ & $0,19297^{\mathrm{ns}}$ & 0,13398 \\
\hline 21 & $2006 / 2007$ & 4 & 13 & 1,836 & $0,06940^{\text {ns }}$ & $0,58756^{*}$ & 0,09845 \\
\hline 22 & $2006 / 2007$ & 3 & 19 & 1,352 & $0,11438^{\text {ns }}$ & $0,69199 *$ & 0,11518 \\
\hline 23 & $2007 / 2008$ & 4 & 15 & 1,513 & $0,19987^{\mathrm{ns}}$ & $0,23320^{*}$ & 0,11840 \\
\hline 24 & $2007 / 2008$ & 3 & 20 & 1,202 & $0,05918^{\mathrm{ns}}$ & $0,32739 *$ & 0,10203 \\
\hline 25 & 2008 & 3 & 20 & 2,127 & $0,05808^{\mathrm{ns}}$ & $0,21262 *$ & 0,07773 \\
\hline 26 & $2008 / 2009$ & 3 & 18 & 0,620 & $0,17270^{*}$ & $0,05952 *$ & 0,02258 \\
\hline
\end{tabular}

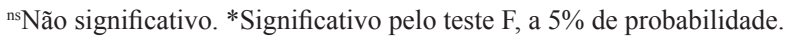


modelo de 76,8 para $83,4 \%$ (Tabela 3 ). A AS, medida de precisão experimental adequada para ensaios com milho (Cargnelutti Filho \& Storck, 2009), feijão e soja (Cargnelutti Filho et al., 2009b), indicou que, em $23 \%$ dos ensaios sem uso do método de Papadakis, a

Tabela 2. Número de casos em que as diferentes hipóteses de nulidade foram rejeitadas, a $5 \%$ de probabilidade, para análise de variância (ANOVA), covariável do método de Papadakis e análise pelo método de Papadakis, em 26 ensaios de competição de cultivares de feijão.

\begin{tabular}{lccc}
\hline Hipótese & ANOVA & Covariável & Papadakis \\
\hline Blocos & $12(46 \%)$ & $13(50 \%)$ & - \\
Cultivares & $23(88 \%)$ & 0 & $25(96 \%)$ \\
Covariável & - & - & $24(92 \%)$ \\
Normalidade $(\mathrm{N})$ & 0 & 2 & 1 \\
Aleatoriedade $(\mathrm{Al})$ & 0 & 0 & 0 \\
Homogeneidade $(\mathrm{H})$ & 0 & 0 & 0 \\
Aditividade (Ad) & 2 & - & - \\
N e Al & 0 & 0 & 0 \\
N e H & 0 & 0 & 0 \\
N e Ad & 0 & - & - \\
Al e H & 0 & 0 & 0 \\
Al e Ad & 0 & - & - \\
H e Ad & 0 & - & - \\
Paralelismo da covariável & - & - & $9^{(1)}$ \\
\hline
\end{tabular}

(1)Número de ensaios com respostas não paralelas para a covariável. precisão foi muito alta ( $\mathrm{AS} \geq 0,90)$. Já com uso deste método, observou-se precisão muito alta em 50\% dos ensaios, o que é indicativo da importância de seu uso para melhoria da precisão experimental. $\mathrm{O}$ uso do método de Papadakis, no presente trabalho e nos ensaios com soja e milho, proporcionou aumento da média da AS e redução da amplitude de variação e do coeficiente de variação desta medida de precisão, isto é, a precisão experimental ficou mais homogênea e mais alta.

Em média, o uso do método proposto foi $2,3 \%$ mais eficiente do que o uso do modelo com delineamento de blocos ao acaso, pois a eficiência relativa do método de Papadakis (ER P, Tabela 3) foi igual a 102,3\%, com variação entre 51,3 e $364,3 \%$ e CV de $60,3 \%$. É importante ressaltar que a violação dos pressupostos dos erros ocorreu em proporções semelhantes com o uso dos dois métodos (Tabela 2). Resultados favoráveis ao uso do método de Papadakis também foram relatados para aplicação no melhoramento genético de feijão e de milho (Souza et al., 2000; Costa et al., 2005).

A média dos coeficientes de regressão linear (efeito da covariável, Beta) foi igual a 1,287, com amplitude de 1,228 (Tabela 3), o que indica que os valores

Tabela 3. Média, amplitude e coeficiente de variação (CV) da produtividade de grãos $\left(\mathrm{Mg} \mathrm{ha}^{-1}\right)$ de diferentes estatísticas estimadas em ensaios de competição de cultivares de feijão, relacionadas à aplicação do método de Papadakis.

\begin{tabular}{|c|c|c|c|c|c|c|}
\hline \multirow[t]{2}{*}{ Estatísticas $^{(1)}$} & \multicolumn{3}{|c|}{ Sem Papadakis } & \multicolumn{3}{|c|}{ Com Papadakis } \\
\hline & Média & Amplitude & CV (\%) & Média & Amplitude & $\mathrm{CV}(\%)$ \\
\hline$\overline{\text { ER (\%) }}$ & 173,8 & 516,8 & 83,6 & - & - & - \\
\hline Média $\left(\mathrm{Mg} \mathrm{ha}^{-1}\right)$ & 1,609 & 2,752 & 43,6 & - & - & - \\
\hline CVe $(\%)$ & 20,3 & 25,7 & 30,3 & 16,3 & 26,3 & 32,5 \\
\hline AS & 0,821 & 0,407 & 12,0 & 0,886 & 0,350 & 8,7 \\
\hline$h^{2}$ & 0,683 & 0,614 & 22,3 & 0,791 & 0,561 & 16,0 \\
\hline D Tukey & 0,882 & 1,127 & 33,2 & 0,715 & 1,029 & 37,3 \\
\hline Amplitude & 1,154 & 1,668 & 37,6 & 1,179 & 1,877 & 39,7 \\
\hline DMS (\%) & 61,4 & 79,7 & 32,1 & 49,4 & 81,7 & 33,8 \\
\hline IDF & 5,95 & 28,1 & 116,2 & 13,62 & 40,94 & 83,7 \\
\hline $\mathrm{R}^{2}(\%)$ & 76,8 & 33,6 & 11,0 & 83,4 & 33,2 & 9,4 \\
\hline Beta & - & - & - & 1,2875 & 1,2280 & 22,8 \\
\hline ER P (\%) & - & - & - & 102,3 & 313,0 & 60,3 \\
\hline$\hat{\rho}_{\mathrm{g}}$ & 0,438 & 0,695 & 38,3 & 0,576 & 0,731 & 30,1 \\
\hline $\mathrm{J}\left(\mathrm{R}^{2}=80 \%\right)$ & 7,3 & 35,3 & 101,1 & 4,0 & 24,2 & 118,4 \\
\hline
\end{tabular}

(1)ER, eficiência relativa dos ensaios com efeito de blocos significativos; CVe, coeficiente de variação do erro experimental; AS, acurácia seletiva; $\mathrm{h}^{2}$, coeficiente de herdabilidade; D Tukey, diferença mínima significativa pelo teste de Tukey, a 5\% de probabilidade; Amplitude, amplitude entre médias; DMS, D Tukey em percentagem da média; IDF, índice de diferenciação de Fasoulas; $\mathrm{R}^{2}$, coeficiente de determinação do modelo; Beta, efeito da covariável coeficiente de regressão linear; ER P, eficiência relativa do uso do método de Papadakis; $\hat{\rho}_{\mathrm{g}}$, coeficiente de repetibilidade; $\mathrm{J}\left(\mathrm{R}^{2}=80 \%\right)$, número de repetições associado à determinação ou à precisão de $80 \%$. 
de produtividade de grãos puderam ser ajustados significativamente em $92 \%$ dos ensaios, para mais ou para menos. Para cada unidade a mais na covariável, o valor da parcela é acrescido em 28,7\% (multiplicado por $1,287)$, valor menor $(1,44)$ do que o obtido para ensaios com soja (Storck et al., 2008) e semelhante $(1,34)$ ao obtido com milho (Storck et al., 2010). Entretanto, para $35 \%$ dos ensaios, a resposta do valor de Beta não foi paralela entre as cultivares (Tabela 2). A falta de paralelismo indica que as cultivares de feijão de um mesmo ensaio têm suas médias ajustadas em intensidades variadas, dependendo do coeficiente de regressão de cada cultivar, em que o ajuste das médias é realizado pelo mesmo coeficiente de regressão para todas as cultivares. A falta de paralelismo, em proporções semelhantes, foi obtida em soja e milho com 49 e $40 \%$, respectivamente (Storck et al., 2008, 2010).

$\mathrm{Na}$ análise de repetibilidade, o valor médio do coeficiente de correlação intraclasse $\left(\hat{\rho}_{\mathrm{g}}\right)$, estimado no método Papadakis, foi de 0,438 , e a redução do número de repetições necessário para uma dada precisão foi expressiva. Ao aplicar o método de Papadakis, foi necessário, em média, apenas a metade do número de repetições para predizer o valor real dos indivíduos (cultivares), com base na precisão pré-estabelecida $\left(\mathrm{R}^{2}=0,80\right)$, quando comparado à análise usual (Tabela 3). Essa redução no número de repetições foi menor do que a obtida em ensaio com a cultura da soja e do milho (Storck et al., 2008, 2010).

Em ensaios de feijão, normalmente são usadas três repetições, valor inferior às sete repetições necessárias para constatar diferenças existentes entre genótipos com confiança de $80 \%$. Contudo, para uma mesma confiança, com uso do método de Papadakis, as três repetições são suficientes, e a determinação ou a precisão seria igual a $\mathrm{R}^{2}=0,803$.

\section{Conclusões}

1. A análise da produtividade de grãos dos ensaios de competição de cultivares de feijão por meio do método de Papadakis mantém adequados os pressupostos de normalidade, aleatoriedade, homogeneidade e aditividade.

2. O método de Papadakis melhora os índices das medidas de precisão experimental e reduz o número de repetições necessário para a predição do desempenho de cultivares de feijão, com um mesmo grau de precisão, em termos de produtividade de grãos.

\section{Agradecimentos}

Ao Conselho Nacional de Desenvolvimento Científico e Tecnológico, pelo apoio financeiro.

\section{Referências}

BANZATTO, D.A.; KRONKA, S. do N. Experimentação agrícola. 4.ed. Jaboticabal: FUNEP, 2006. 237p.

CANDIDO, L.S.; PERECIN, D.; LANDELL, M.G. de A.; PAVAN, B.E. Análise de vizinhança na avaliação de genótipos de cana-de-açúcar. Pesquisa Agropecuária Brasileira, v.44, p.1304-1311, 2009.

CARGNELUTTI FILHO, A.; RIBEIRO, N.D.; STORCK, L. Número de repetições para a comparação de cultivares de feijão. Ciência Rural, v.39, p.2419-2424, 2009a.

CARGNELUTTI FILHO, A.; RIBEIRO, N.D.; STORCK, L.; JOST, E.; POERSCH, N.L. Tamanho de amostra de caracteres de cultivares de feijão. Ciência Rural, v.38, p.635-642, 2008.

CARGNELUTTI FILHO, A.; STORCK, L. Estatísticas de avaliação da precisão experimental em ensaios de cultivares de milho. Pesquisa Agropecuária Brasileira, v.42, p.17-24, 2007.

CARGNELUTTI FILHO, A.; STORCK, L. Medidas do grau de precisão experimental em ensaios de competição de cultivares de milho. Pesquisa Agropecuária Brasileira, v.44, p.111-117, 2009.

CARGNELUTTI FILHO, A.; STORCK, L.; LÚCIO, A.D. Ajustes de quadrado médio do erro em ensaios de competição de cultivares de milho pelo método de Papadakis. Pesquisa Agropecuária Brasileira, v.38, p.467-473, 2003.

CARGNELUTTI FILHO, A.; STORCK, L.; RIBEIRO, N.D. Medidas da precisão experimental em ensaios com genótipos de feijão e de soja. Pesquisa Agropecuária Brasileira, v.44, p.1225-1231, 2009b.

COSTA, J.R.; BUENO FILHO, J.S. de S.; RAMALHO, M.A.P. Análise espacial e de vizinhança no melhoramento genético de plantas. Pesquisa Agropecuária Brasileira, v.40, p.1073-1079, 2005.

CRUZ, C.D.; REGAZZI, A.J. Modelos biométricos aplicados ao melhoramento genético. 2.ed. Viçosa: UFV, 1997. 390p.

FASOULAS, A.C. Rating cultivars and trials in applied plant breeding. Euphytica, v.32, p.939-943, 1983.

GÓMEZ, K.A.; GÓMEZ, A.A. Statistical procedures for agricultural research. $2^{\text {nd }}$ ed. New York: John Wiley, 1984. 680p.

MARQUES, D.G.; STORCK, L.; LOPES, S.J.; MARTIN, T.N. Qualidade dos ensaios de competição de cultivares de milho no Estado do Rio Grande do Sul. Ciência Rural, v.30, p.381-385, 2000.

PAPADAKIS, J.S. Méthode statistique pour des expériences sur champ. Thessalonike: Institut d'Amélioration des Plantes à Salonique, 1937. 30p. (Bullettin, 23). 
RAMALHO, M.A.P.; FERREIRA, D.F.; OLIVEIRA, A.C. de. Experimentação em genética e melhoramento de plantas. 2.ed. Lavras: UFLA, 2005. 322p.

RESENDE, M.D.V. de; DUARTE, J.B. Precisão e controle de qualidade em experimentos de avaliação de cultivares. Pesquisa Agropecuária Tropical, v.37, p.182-194, 2007.

RIBEIRO, N.D.; STORCK, L.; MELLO, R.M. Bordadura em ensaios de competição de genótipos de feijoeiro relacionados à precisão experimental. Ciência Rural, v.31, p.13-17, 2001.

SEBER, G.A.F. Linear regression analysis. NewYork: John Wiley, 1976. 465p.

SNEDECOR, G.W.; COCHRAN, W.G. Statistical methods. $8^{\text {th }}$ ed. Ames: Iowa State University Press, 1989. 503p.

SOUZA, E.A. de; GERALDI, I.O.; RAMALHO, M.A.P. Alternativas experimentais na avaliação de famílias em programas de melhoramento genético do feijoeiro. Pesquisa Agropecuária Brasileira, v.35, p.1765-1771, 2000.

SPRENT, P.; SMEETON, N.C. Applied nonparametric statistical methods. $4^{\text {th }}$ ed. Boca Raton: Chapman \& Hall, 2007. 530p.

STEEL, R.G.D.; TORRIE, J.H.; DICKEY, D.A. Principles and procedures of statistics: a biometrical approach. 3.ed. New York: McGraw Hill Book, 1997. 666p.

STORCK, L.; LOPES, S.J.; CARGNELUTTI FILHO, A.; LÚCIO, A.D.; TOEBE, M. Experimental precision in corn trials using the Papadakis method. Ciência e Agrotecnologia, v.34, p.1458-1464, 2010.

STORCK, L.; STECKLING, C.; ROVERSI, T.; LOPES, S.J. Utilização do método de Papadakis na melhoria da qualidade experimental de ensaios com soja. Pesquisa Agropecuária Brasileira, v.43, p.581-587, 2008.

Recebido em 28 de janeiro de 2011 e aprovado em 29 de julho de 2011 